\title{
Impact of rotavirus infection on a paediatric hospital in the East End of London
}

\author{
J S Noel, S P Parker, K Choules, A D Phillips, J Walker-Smith, W D Cubitt
}

\begin{abstract}
Aims-To study the impact of confirmed rotavirus infection at a paediatric hospital; to use the data to obtain a minimum estimate of the cost of treating reported cases of rotavirus in England and Wales. Methods-Data were obtained on all patients with rotavirus over a two year period. Information was collected on 386 patients with rotavirus infection who were treated at the 120 bed Queen Elizabeth Hospital for Children in East London. This included the virus serotype, the patient's age, whether they required intravenous infusion, duration of hospital stay, numbers of patients treated in the casualty department, and numbers who had to be admitted. Treatment costs were obtained from the Finance Department of the Hospitals for Sick Children.
\end{abstract}

Results-The minimum cost of treating patients, excluding the cost of medical staff at the hospital, was estimated to be $£ 95400$ a year. One hundred and forty eight $(38 \%)$ patients were admitted to the wards and a further 49 patients developed symptoms while in hospital. Intravenous infusion was required by 18 patients. The mean duration of hospital stay was 5.5 days. One hundred and eighty nine $(49 \%)$ patients were treated with oral rehydration solution in casualty, given advice, and sent home. Ninety four per cent of the patients were aged under 2 years. The findings were comparable with those obtained in a study at Texas Children's Hospital, USA. The G serotype (VP7) of rotavirus did not influence the severity of infection.

Conchusion-Rotavirus infections accounted for a significant number of patients treated in casualty, admissions to hospital, and bed occupancy in a paediatric hospital. The estimated cost of treating reported cases of rotavirus in England and Wales is in excess of $£ 6.3$ million a year.

(F Clin Pathol 1994;47:67-70)

Although human rotavirus (HRV) has been established as a major cause of diarrhoeal disease in children throughout the world, there have been few attempts to assess the impact of such infections on the Health Service. The most comprehensive survey to date was carried out over a 10 year period at a large paediatric hospital in Texas, USA. ${ }^{1}$ The present study was designed to examine the impact of HRV infections on a 120 bed paediatric hospital in the East End of London and to compare our results with those obtained in the study at Texas Children's Hospital (TCH). The results could then be used to obtain a minimum estimate for the cost of treatment of reported HRV infections in England and Wales each year.

\section{Methods}

The survey was conducted from July 1990 through June 1992 at Queen Elizabeth Hospital for Children in East London. The hospital has 120 beds and draws $93 \%$ of its patients from the North East Thames Regional Health Authority. Most admissions for gastroenteritis come from Tower Hamlets and City and Hackney health districts which have a high immigrant population, urban deprivation, and where there are approximately 6900 births a year.

During the period, 3777 faecal samples from children at the Queen Elizabeth Hospital for Children (QEH) were examined at the Department of Virology, the Hospitals for Sick Children. Faecal extracts were made directly in negative stain, $2 \%$ potassium phosphotungstic acid ( $\mathrm{pH} 6 \cdot 4$ ), and examined in a Philips 201 electron microscope for the presence of virus particles. Samples found to contain $\mathrm{HRV}$ were stored at $4^{\circ} \mathrm{C}$ and serotyped as described before, ${ }^{2}$ with an indirect enzyme immune assay (EIA), using cross reactive polyclonal antisera to group A HRV as capture antibodies and G type VP7 specific monoclonal antibodies ${ }^{3}$ as detectors.

Epidemiological data on the numbers and seasonal distribution of rotavirus infections in England and Wales were obtained from the Public Health Laboratory Service, Communicable Disease Surveillance Centre (CDSC) reports.

Since 1989 data on patients attending the gastroenteritis clinic at $\mathrm{QEH}$ have been recorded on computer. This contains relevant hospital record information relating to the following: percentage dehydration on admission; whether intravenous saline infusion was required; and admission and discharge data. Information on the age, sex, location of specimens and dates when virological tests were performed were also available.

Estimates of costs of treating patients who had attended the gastroenteritis clinic at 
QEH as outpatients or inpatients were provided by the Finance Department, Hospitals for Sick Children, Special Health Authority. The average daily cost of treating a patient in casualty, including the average drug costs, was $£ 37$, and the average cost for a patient admitted to the infectious diseases ward was $£ 146$ a day. The cost of laboratory investigations, which include bacteriology, virology, and biochemistry, was estimated at $£ 64.58$ a patient. Data on bed occupancy and numbers of patients treated at QEH between April 1989 and May 1991 were obtained from figures published in the hospital's annual report (1990-1).

\section{Results}

The results of the electron microscopic examination of 3777 faecal samples for the two year period at $\mathrm{QEH}$ were compared with reports from England and Wales (fig 1). HRV was detected in $545(14 \cdot 4 \%)$ samples obtained from 386 patients. The HRV cases treated at QEH accounted for $386(1 \cdot 2 \%)$ out of 31381 reports recorded by CDSC over the two year period. Most HRV cases at QEH and throughout England and Wales occurred

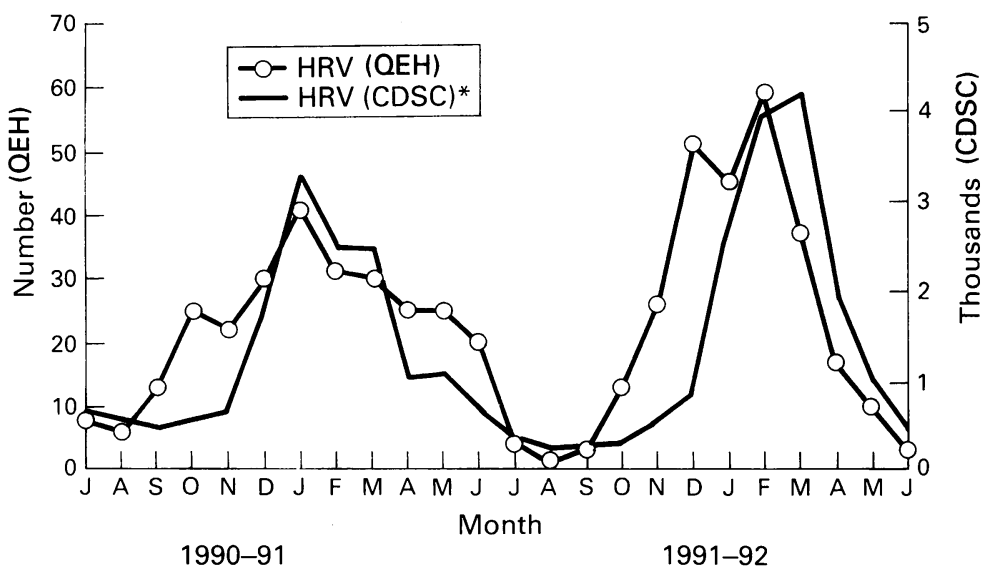

*CDSC data provided with kind permission of the editor

Figure 1 Seasonal distribution of human rotavirus infection at Queen Elizabeth Hospital and cases reported from England and Wales, Fuly 1990-Fune 1992. during the cooler months of the year. (October-March) (fig 1).

The results of serotyping HRV infections in 937 patients at QEH over the period 1984-90 have been published. ${ }^{2}$ The distribution of $\mathrm{G}$ serotypes occurring during 1990-2 are shown in fig 2 . In 1989-90 serotype G4 became the predominant serotype, but the situation reverted to serotype G1 being the dominant type throughout the period under study (July 1990 to June 1992). Types G2, G3, and G4 were detected with varying frequency throughout the period. Serotype G8 was encountered for the first time in 1991 (two cases); a further three cases were found in 1992.

Data were available on the age of 368 of the 386 children with HRV (fig 3). Three hundred and forty five (93\%) of the infections were in children under 2 years, among whom were 41 infants aged under 4 months. Infection was sufficiently severe to necessitate admission to hospital in $17(41 \%)$ patients in this age group; two patients aged 2 months required intravenous infusion. A further nine cases were believed to be hospital acquired as they had been admitted more than three days before the onset of diarrhoea.

Only 18 of $386(5 \%)$ patients required intravenous infusion; 15 cases were due to serotype $\mathrm{G} 1$, two to serotype $\mathrm{G} 4$, and one to serotype G2. The five patients infected with serotype G8 were all treated with oral rehydration solutions in the casualty unit.

An estimate of the cost of treating 337 patients with confirmed HRV infection at QEH are presented in fig 4. Many of the patients who attended the casualty unit were given oral rehydration fluids, advice on how to use them, and told to return if symptoms did not resolve. Those with more severe dehydration (148 of $337,44 \%$ ) were admitted to the infectious diseases ward where isolation facilities are available.

There was no evidence of a nosocomial outbreak of HRV infection during the period of the study, but a further 49 sporadic cases occurred on four of the other wards in the hospital. Thirty one of these were on a general paediatric ward which treats patients under 2 years of age. Many of these cases
$1989-90$

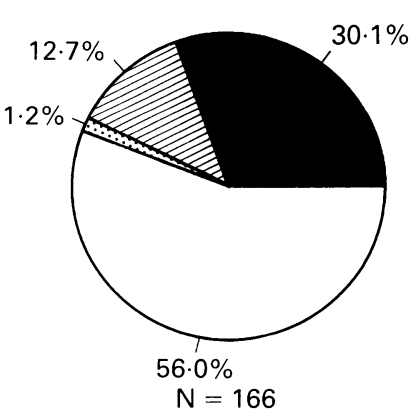

$1990-91$

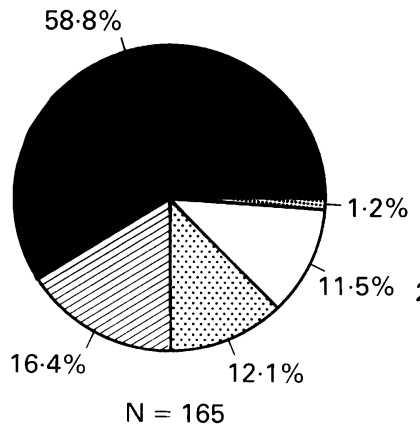

$1991-92$

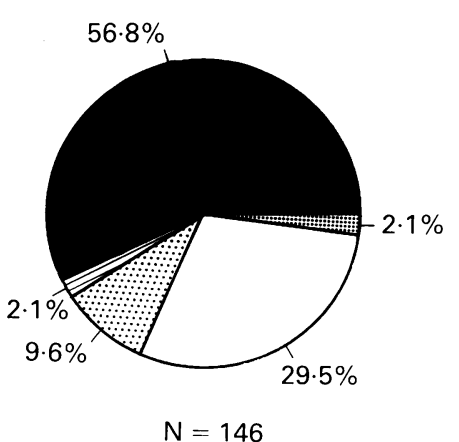

HRV serotype

$1 \square 2 \square 3 \square 4$ 橧 $8 \quad N=$ Number tested

Figure 2 Distribution of G (VP7) human rotavirus serotypes at Queen Elizabeth Hospital, 1989-92. 


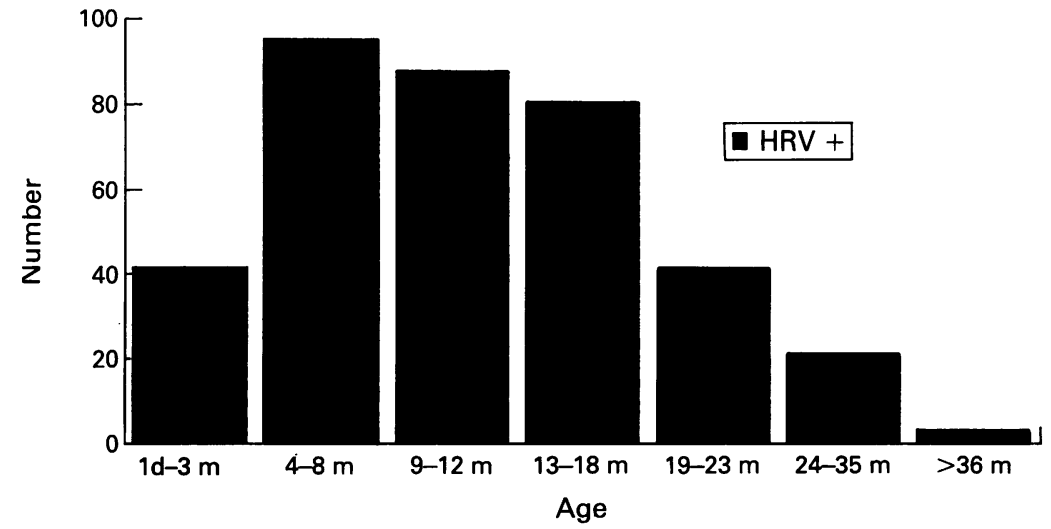

Figure 3 Age distribution of 368 patients with rotavirus infection treated at Queen Elizabeth Hospital, fuly 1990-fune 1992. resulted in a prolonged stay in hospital and a reduction in the availability of beds.

Detailed information was readily available on the database for 118 of 148 patients admitted to the observation ward. The total number of bed days was 654 , with a mean (SD) duration of stay of $5 \cdot 5(4 \cdot 5)$ days. This represented $1.3 \%$ (148 of 12300 ) of the admissions and $1.2 \%$ of the inpatient days at $\mathrm{QEH}$ during the study period. The estimate of the cost of treating the 118 patients admitted to the observation ward was $£ 103104$ and for the 189 treated in the casualty unit, $£ 19198$. On the assumption that the average hospital stay associated with HRV infection for the other 79 inpatients with HRV infection was also 5.5 days, the additional cost of treating them would have been $£ 68538$. This gives an overall estimate of $£ 190840$ (£95 420 a year).

\section{Discussion}

The results confirm previous observations made throughout the world that HRV is a major cause of admissions to hospital of children aged under 2 years for treatment of diarrhoea. ${ }^{4}$ Most cases at QEH and throughout England and Wales occur during the last six months of the financial year when hospital budgets are often under immense strain. This is compounded by the fact that the peak of admissions of children with bronchiolitis due to respiratory syncytial virus occurs at the same time. Our findings indicated that $2 \cdot 7 \%$ of all admissions (casualty plus the infectious diseases ward) to QEH were due to HRV. This is remarkably similar to the findings $(2 \cdot 2 \%-4 \cdot 6 \%)$ of a 10 year study on the impact of rotavirus infection at the 328 bed Texas Children's Hospital. ${ }^{1}$ It is frequently stated that HRV infections in infants aged up to 3 months are either asymptomatic or mild ${ }^{56}$ but in the present survey and at the Texas Hospital, a significant number of the patients requiring hospital treatment were in this age group, $(7.0 \%$ and $13.3 \%$, respectively). In two patients (both aged 2 months) admitted to QEH dehydration was severe, necessitating intravenous infusion, and in a previous outbreak on a special care baby unit in another hospital, several deaths occurred due to HRV (Cubitt and Holzel, unpublished data).

The mean duration of hospital stay for patients with HRV was 5.5 days at QEH and 5.3 days at the Texas Children's Hospital. Further comparison can be made of the costs of treating inpatients at the two hospitals. At QEH this was estimated to be $£ 158$ a day, excluding the cost of medical staff, and at Texas Children's Hospital US\$603, based on the average revenue per operating bed. The minimum cost, based on treating 386 patients with HRV over 2 years at QEH is estimated at $£ 95420$ a year compared with $\geqslant U S \$$ 1500000 to treat 473 patients a year at the 328 bed children's hospital in the USA.

In 1991,13260 cases of HRV were reported to the CDSC by laboratories in England and Wales. Most of these cases were from patients treated in hospital and therefore do not take into account the large number of cases treated by general practitioners. The extent of the problem in the general community was shown by a study in the USA in which it was estimated that 25 work or school days per 100 children are lost each year and

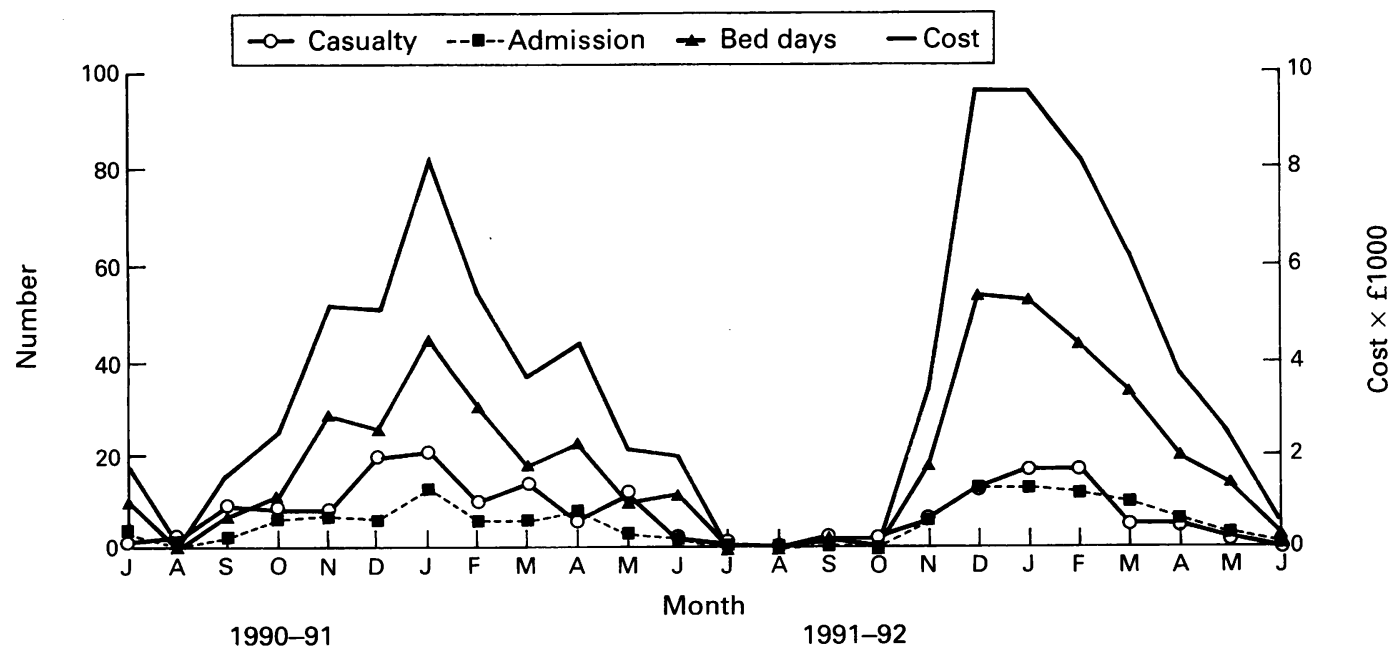

Figure 4 Impact of human rotavirus at Queen Elizabeth Hospital, fuly 1990-fune 1992. 
that $14 \%$ of all children are treated by a doctor for HRV associated diarrhoea. ${ }^{7}$ Extrapolating from our estimates in the present study, in which $49 \%$ of the patients were treated in hospital and $51 \%$ in casualty, the cost (excluding cost of medical staff) of treating the 13260 confirmed HRV cases is in excess of $£ 6.3$ million a year. It has to be borne in mind that these represent only a minute fraction of HRV infections occurring in the community as a whole.

It is evident from these limited studies that the true cost of HRV infections to health services is enormous. This illustrates the need for continued research and dissemination of knowledge about oral rehydration solutions so that children can be treated at home or by general practitioners rather than being admitted to hospital. This may prove to be a more cost effective strategy than vaccination against HRV. As there is an increasing emphasis on the cost of health provision in the United
Kingdom there is a need for an indepth study to determine the true impact of HRV in the community at large.

1 Matson DO, Estes MK. Impact of rotavirus infection at a large pediatric hospital. F Infect Dis 1990;162:598-604.

2 Noel JS, Beards GM, Cubitt WD. Epidemiological survey of human rotavirus serotypes and electropherotypes in young children admitted to two children's hospitals in London from 1984 to 1990 . 7 Clin Microbiol 1991;29: 2213-19.

3 Coulson BS, Unicomb LE, Pitson GA, Bishop RF. Simple and specific enzyme immunoassay using monoSimple and specific enzyme immunoassay using monoclonal antibodies for serotyping human rotaviruses. $f$ .

4 Kapikian AZ, Chanock RM. Viral gastroenteritis. In: A S Evans, ed. Viral infections of humans. New York: Plenum Press, 1989:293-340.

5 Maldonado YA, Yolken RH. Rotavirus. In: Farthing MJ, ed. Clinical gastroenterology. Virus infections of the gut and liver. London: Baillière Tindall, 1990:609-25.

6 Flores J, Kapikian AZ. Rotavirus vaccines. In: Farthing MJ, Keusch GT, eds. Enteric infection. Mechanisms, manifestations and management. London: Chapman and Hall, 1989:509-27.

7 Centers for Disease Control. Viral agents of gastroenteritis: public health importance and outbreak management. Morbidity and Mortality Weekly Report 1990; 39(RR-5): 1-24 